\title{
engineering applications
}

3 Gang Wu ${ }^{1}$, Qiang Yan $^{2}$, Andrew Jones ${ }^{3,5}$, Yinjie J. Tang ${ }^{1, \dagger}$, Stephen S Fong ${ }^{2, \Theta}$, Mattheos

1. Department of Energy, Environmental and Chemical Engineering, Washington University in

$7 \quad$ St. Louis, St. Louis, MO, USA

8 2. Department of Chemical and Life Science Engineering, Virginia Commonwealth University,

9 Richmond, Virginia 23284-3028

10 3. Department of Chemical and Biological Engineering, Rensselaer Polytechnic Institute, Troy,

11 NY 12180

12 4. Department of Biological Sciences, Rensselaer Polytechnic Institute, Troy, NY 12180

13 5. Center for Biotechnology and Interdisciplinary Sciences, Rensselaer Polytechnic Institute,

14 Troy NY 12180

15

16

17 Corresponding:

$18{ }^{\dagger}$ :Email: yinjie.tang@wustl.edu; Phone: (314)-935-3441

$19{ }^{\Theta}$ :Email: ssfong@ vcu.edu; Phone: (804) 827-7038

20 *:Email: koffam@ rpi.edu; phone: (518)-276-2220 


\section{Abstract}

22 Engineering cell metabolism for bio-production not only consumes building blocks and energy

23 molecules (e.g., ATP) but also triggers energetic inefficiency inside the cell. The metabolic

24 burdens on microbial workhorses lead to undesirable physiological changes, placing hidden

25 constraints on host productivity. We discuss cell physiological responses to metabolic burdens,

26 as well as strategies to identify and resolve the carbon and energy burden problems, including

27 metabolic balancing, enhancing respiration, dynamic regulatory systems, chromosomal

28 engineering, decoupling cell growth with production phases, and co-utilization of nutrient

29 resources. To design robust strains with high chances of success in industrial settings, novel

30 genome-scale models (GSMs), ${ }^{13} \mathrm{C}$-metabolic flux analysis (MFA), and machine learning

31 approaches are needed for weighting, standardizing and predicting metabolic costs.

33 Key words: ${ }^{13} \mathrm{C}-\mathrm{MFA}$, genome-scale model, machine learning, chromosomal engineering 


\section{Opportunities and challenges in synthetic biology (SynBio) applications}

46 Metabolic engineering has created diverse microbial cell factories for applications in

47 food, pharmaceutical, biofuel, and commodity chemical synthesis. Initially, industrial microbes

48 were developed by random mutations or by expressing only one or two new enzymes. In the past

49 decade, new gene sequencing/synthesis/editing techniques have allowed for complex genetic

50 manipulations to build new cellular functions, and the International Genetically Engineered

51 Machine (iGEM) competition promoted the concept of using standard modules (e.g., synthetic

52 pathway and genetic circuits) for facilitating bio-production [1,2]. Despite great technological

53 advances, engineered microbial platforms cannot cheaply manufacture products due to poor

54 production titer, rate and yield [3,4]. Moreover, engineered microbes often show unpredictable or

55 unstable physiology, and thus iterative "build-design-test-learn" cycles have to be employed for

56 strain improvement [5]. In March 2015, representatives from industry, academic institutions, and

57 NIST addressed a key issue preventing synthetic biology from reaching its potential: "Unlike

58 silicon-based electronic devices, synthetic organisms assembled from genetic components do not

59 always have predictable properties" [6].

60 When a complex pathway is introduced into the host but shows low performance, one

61 perspective is that the new pathway has "intermediate toxicity" or "low enzyme activity".

62 Therefore, strain improvement often focuses on searching for "bottleneck" steps and tuning

63 enzyme functions. Another possibility for low productivity in engineered strains is metabolic

64 burden, a long-standing problem in biotechnology. Metabolic burden (see Glossary) is defined

65 as the portion of a host cell's resources - either energy molecules (e.g., NAD(P)H and ATP) or

66 carbon building blocks - that is used to construct and operate engineered pathways [7]. Since

67 intracellular carbon and energy resource distribution in healthy cells have been "optimized" by 
68 natural evolution, hijacking cell resources for pathway overexpression, plasmid maintenance,

69 and product synthesis may upset normal cellular processes.

70 Metabolic engineers first noticed metabolic burden. With the advance of recombinant

71 DNA technology in the 1970s and 1980s, overexpression of proteins to produce desired products

72 has been attempted, which often results in reduced cell growth and increased mutation rates [7-

73 9]. Metabolic burden could also cause inefficiency in the cell ATP supply, affecting broad

74 cellular functions. A typical example is that Azotobacter vinelandii transformed with plasmids

75 gradually lost its ATP-dependent $\mathrm{N}_{2}$ fixation ability when the plasmid copy number increased

$76[7,10]$. In yet another case, Pseudomonas putida completely lost its ability to synthesize

77 siderophores after transformation with high copy plasmids [11]. Therefore, metabolic burden is a

78 key factor leading to undesired physiological changes, and the cost/benefit of synthetic biology

79 strategies needs to be evaluated under the light of metabolic burdens.

80

81 A "cliff" of host productivity under metabolic burden

ATP powers all cellular functions and cells must produce sufficient ATP for both

83 biosynthesis requirements and cellular maintenance. However, biological/physical factors restrict

84 the capabilities of cellular energy metabolism [12]. For example, the ratio of cell membrane

85 surface area to cell volume influences the overall mass exchange efficiency and the upper limit

86 of nutrient and oxygen uptake rates [13]. For E. coli cultures [14], upper bound glucose uptake

87 rates are $\sim 18 \mathrm{mmol} / \mathrm{gDW} / \mathrm{h}$ (gDW: grams of dry weight) in anaerobic conditions and 11

$88 \mathrm{mmol} / \mathrm{gDW} / \mathrm{h}$ in aerobic conditions with 5.3 ATP molecules per glucose consumed formed in

89 aerobic conditions compared with 1.96 ATP per glucose in anaerobic conditions. The nutrient

90 uptake places a hard constraint on cell catabolic rates. Moreover, ATP synthesis is a 
91 thermodynamically inefficient process (part of the substrate energy will be lost as heat) [15].

92 Under optimal conditions, oxidation of one NADH generates 2.5 ATP (i.e., P/O=2.5) [15]. For

93 engineered strains, proton gradients are dissipated through the cell membrane instead of charging

94 ATP synthase, causing poor P/O ratios $(<2)$ [16-18]. Therefore, cell physiology and bio-

95 production are particularly sensitive to metabolic burden from ATP consumption.

96 To demonstrate the highly nonlinear impact of energy burden on biosynthesis, flux

97 balance analysis (FBA) was used with a constraint-based model to simulate the adverse impacts

98 of ATP metabolism on E. coli fatty acid, isobutanol and acetate yields (Figure 1) [19]. The

99 simulations showed that native cell metabolism can withstand a certain amount of ATP loss

100 without having apparent biosynthesis deficiency (i.e., forming a "plateau"). If cell metabolism

101 demands more ATP, cells can utilize respiration (increase oxygen influx) to maintain their well-

102 being. When catabolism and respiration become insufficient to accommodate the further increase

103 of ATP expenditure, the biosynthesis yield will suddenly drop, forming a "cliff" towards the

104 "death valley" (production yields drop to minimal levels). When a strain's metabolic status is on

105 the "cliff", it may show unstable performance and become highly sensitive to suboptimal growth

106 conditions (such as low oxygen level). Moreover, three products show differences in the yield

107 plateau phenomenon. Fatty acid synthesis consumes both NADPH and ATP. Cell productivity is

108 sensitive to oxygen supply, maintenance loss, and P/O ratios (Figure 1A and 1B). Isobutanol

109 synthesis only consumes $\mathrm{NAD}(\mathrm{P}) \mathrm{H}$, and it can be produced in the non-growth phase due to

110 competition with amino acid biosynthesis. Therefore, the area of the "death valley" in Figure 1C

111 and 1D (showing isobutanol production) is reduced compared with fatty acid production,

112 suggesting isobutanol has a higher chance of stable industrial production. Acetate synthesis has 
113 net ATP generation; its production has the largest yield plateau and thus is more likely to achieve

114 robust production.

\section{Use of flux analysis to precisely measure metabolic burdens}

117 Among systems biology tools, fluxomic measurements can provide direct knowledge of

118 metabolic conversion of carbon sources into products. It is the only tool to understand the

119 allocations of cell resources during biosynthesis. FBA uses the stoichiometry of biochemical

120 reactions in addition to the measurement of inflow (uptake) fluxes to predict cellular phenotypes

121 and biosynthesis yields. FBA can characterize cell energy metabolism by dividing maintenance

122 costs into two categories [18]: non-growth associated maintenance (NGAM) by "resting"

123 cells, and growth associated maintenance (GAM). Due to the size and underdetermined nature

124 of metabolic networks, FBA has a high degree of uncertainty and requires constraints to analyze

125 the solution spaces. ${ }^{13} \mathrm{C}$ tracing can assist FBA to rigorously measure functional pathways

126 throughout the metabolic network (which is called ${ }^{13} \mathrm{C}$-metabolic flux analysis, or ${ }^{13} \mathrm{C}-\mathrm{MFA}$ ).

$127{ }^{13} \mathrm{C}-\mathrm{MFA}$ can validate the function of genes or genetic circuits, provide knowledge on bottleneck

128 nutrient sources, identify metabolic engineering targets, and directly quantify energy flows (i.e.,

129 ATP and cofactor generations and consumptions). ${ }^{13} \mathrm{C}-\mathrm{MFA}$ is widely used to identify specific

130 flux changes in the network of such mutants.

131 During pathway engineering, cell resource over-expenditure for heterologous enzyme

132 synthesis can trigger the re-organization of carbon fluxes and reduce cell well-being [20]. To

133 identify "the straw that broke the camel's back," ${ }^{13} \mathrm{C}-\mathrm{MFA}$ in combination with

134 transcriptomics/proteomics and genome-scale modeling can provide a comprehensive

135 understanding of cell responses to metabolic burdens at different cellular levels (from 
136 transcriptome to fluxome). ${ }^{13} \mathrm{C}-\mathrm{MFA}$ is particularly useful to link these synthetic biology

137 components (such as transcriptional regulators) to cell functional outputs. In general, gene

138 expression may have an unpredictable impact on the distribution of fluxes because central

139 metabolic fluxes are rarely regulated at the expression level alone [21]. For example, ${ }^{13} \mathrm{C}-\mathrm{MFA}$

140 and transcriptional analysis for fatty acid production in E. coli using overexpression of the free

141 fatty acid pathway and fatty acid transcription regulator revealed complex fluxome responses to

142 fatty acid overproduction, including high ATP maintenance loss, decreased acetate flux,

143 enhanced NADPH producing pathway (pentose phosphate pathway and Entner-Doudoroff

144 pathway), and strong transhydrogenation activity to achieve cofactor balance [22].

145 In the synthetic biology field, ${ }^{13} \mathrm{C}-\mathrm{MFA}$ has not become a routine tool because of two

146 bottlenecks. First, ${ }^{13}$ C-MFA based on amino acid labeling cannot probe cell metabolism in a rich

147 medium, for non-growing cells, or through a genome-scale metabolic network. To resolve such

148 problems, innovative genome-scale MFA and isotopically nonstationary MFA will assist

149 synthetic biology to gain fast snapshots of cell metabolism for improving strain performance [23-

150 25]. However, the time required to analyze the samples and process the data to calculate fluxes

151 through complex metabolism is still long (often taking months for an experienced lab to quantify

152 cell metabolism correctly), and this method cannot satisfy a fast turnover cycle during build-

153 design-test-learn. Second, ${ }^{13}$ C-MFA focuses on global fluxes rather than the activity of

154 individual enzymes. Many synthetic biology hosts only achieve productivity at $\mathrm{mg} / \mathrm{L}$ levels, and

155 the priority is thought to improve the heterologous enzyme activities in the production pathway.

156 From this aspect, ${ }^{13} \mathrm{C}-\mathrm{MFA}$ is useful when the strain performance is close to industrial

157 performance levels.

158 Synthetic biology methods to overcome metabolic burden 
In traditional fermentation engineering, metabolic burden can be minimized through

160 well-controlled bioreactor conditions (such as $\mathrm{pH}$ and oxygen) as well as the use of different

161 media (including yeast extract or other intermediates to boost cell metabolism). Early metabolic

162 engineers also relied on random mutations or adaptive evolution for improved synthesis of

163 growth-associated products, leveraging natural selection for reducing metabolic burden. For

164 example, E. coli strain can be "trained" to have fast growth using lactate: over 250 generations of

165 evolution, its pathway capacity can be improved, especially for these flexible metabolic nodes

166 (such as phosphoenolpyruvate, oxaloacetate, and acetyl-coenzyme A) [26]. In addition, co-

167 cultures can also be a possible solution that divides a biosynthesis pathway into two sections then

168 use two species to share the metabolic burdens.

169 When synthetic biology tools became available, they allowed more elegant strategies to 170 control cell metabolism at different cellular levels to reduce metabolic burden. Transcriptional

171 regulators have attracted interest since alteration of some regulators may alleviate metabolic

172 repressions and unlock cell carbon and energy fluxes [27]. Catabolite repression is present in

173 various microorganisms and influences carbon, energy and cofactor metabolism in response to

174 carbon sources. For example, glucose is the preferred carbon source in E. coli. Deletion of

175 catabolite repression regulators and overexpression of genes required for pentose metabolism

176 allows cell fast growth using glucose and xylose simultaneously [28]. Moreover, Smolke and

177 Keasling studied the effect of mRNA stability and DNA copy number on protein production and

178 discovered the correlations among DNA, mRNA and protein levels are strongly nonlinear [29].

179 Their results indicate that optimally tuning the plasmid copy number and mRNA processing is

180 essential for effective pathway construction. Currently, synthetic biology has already offered

181 broad strategies to reduce energy burdens. Table 1 shows the common strategies to reduce 
182 metabolic burden and balance cell bio-production, including copy number optimization,

183 transcriptional optimization, translational optimization, post-translational optimization, dynamic

184 balancing, compartmentalization, and co-culture strain ratio optimization.

185 In synthetic biology, transcriptional/protein-level regulation of cell production has become a 186 focal point. Among these strategies, dynamic sensors and regulatory systems (DSRSs) employ

187 a biosensor to detect the level of metabolite intermediate and control enzyme expressions, which 188 may be useful to prevent the biosynthesis of unnecessary RNAs/proteins/metabolites and 189 increase the efficiencies of energy and carbon usage [56,57,61]. Unlike static control, DSRSs can

190 promote or repress pathways according to cell growth conditions or intermediate metabolite 191 concentrations. Thereby, DSRSs may also be used to decouple cell biomass growth and

192 production phases (e.g., quorum sensing) so that cell resources can be focused on one major task

193 at a time. Recently, CRISPR systems have been implemented for both chromosomal engineering

194 and systematic down-regulation of gene expression [62] (drawbacks of plasmid engineering

195 include instability and burdens due to DNA/enzyme synthesis). Over the long term, synthetic

196 biology may re-program the entire cell genome to create a "minimal or smart" cell of best energy

197 fitness [63-65].

199 Promotion of metabolic capability in microbial chassis

200 Synthetic biology has attempted to reduce metabolic burden by removing unnecessary

201 genes from microbial chassis. However, such efforts did not show significant benefits. For

202 example, genome reduction of 332 dispensable genes in Bacillus subtilis does not affect the

203 cell's fluxome [66]. This result is consistent with the fact that the DNA synthesis accounts for a

204 very small cell resource usage. In contrast, cell energy metabolism has shown thermodynamic 
constraints to both biomass growth and bio-productivity, which are often limited and difficult to

206 improve. For heterotrophic microbes, ATP generation is coupled with cell catabolism and the

207 outputs from ATP synthase is limited by the cell membrane space. In addition, synthetic biology

208 components may interfere with the proton motive force and reduce ATP generation capabilities

209 [67]. Therefore, improving intracellular energy generation and catabolic metabolism allow the

210 hosts to carry more synthetic biology modules. Three directions can be pursued.

211 First, ATP is mainly synthesized through oxidative phosphorylation in aerobic

212 metabolism. However, respiration rates in many strains are far below theoretical maxima in

213 bioreactors. The respiration efficiency can be successfully increased using the Vitreoscilla

214 hemoglobin, which was first recombinantly expressed in E. coli [68]. This enzyme can improve

215 oxygen membrane transfer and the $\mathrm{P} / \mathrm{O}$ ratio and reduce waste byproducts. Moreover, Zamboni

216 and Sauer enhanced riboflavin production in Bacillus subtilis by knockout of

217 cytochrome $b d$ oxidase to reduce cell ATP maintenance costs [69]. Engineering of respiration

218 may apply to species with several sets of respiratory chains with different efficiencies [70,71]

219 and successful application of this strategy is also closely related to other factors, such as oxygen

220 concentration and medium composition [72].

221 Second, photosynthetic microorganisms convert $\mathrm{CO}_{2}$ to useful products with light as the

222 energy source [73]. However, the photoautotrophic process has been hindered by $\mathrm{CO}_{2}$ and light

223 availability inside of photobioreactors, which leads to low cell density and cost-inefficient

224 harvesting. To circumvent these problems, a photomixotrophic strategy is advantageous [74].

225 Under conditions of sufficient light and glucose, microalgae can consume both $\mathrm{CO}_{2}$ and glucose

226 for biomass production, which potentially leads to higher biomass density. The ability to utilize

227 light as an energy source can be enabled for non-photosynthetic species through heterologous 
228 expression of proteorhodopsins [75-77]. Proteorhodopsins are a class of membrane proteins

229 distributed in a considerable number of species ranging from bacteria to archaea and fungi [78].

230 They can absorb energy from light of different wavelengths and generate a proton gradient used

231 for ATP synthesis. Compared with photosystems, a distinctive advantage for proteorhodopsin is

232 its simplicity for heterologous photosynthesis [79]: the function of proteorhodopsin relies on a

233 single protein with 249 amino acids. Hence, the metabolic costs associated with heterologous

234 expression of proteorhodopsins will be much smaller than intact photosystems. An added

235 potential benefit is that species with proteorhodopsin have demonstrated increased tolerance to

236 environmental stress [75,77].

237 Third, $\mathrm{H}_{2}$ can be utilized by a broad range of microbes. However, using hydrogen as the

238 energy supplier in industrial fermentations is not preferred. This is due to various undesirable

239 properties of $\mathrm{H}_{2}$ such as its low solubility, volatility, and explosiveness. Compared with $\mathrm{H}_{2}$,

240 formate is a better source of energy supply in terms of uptake efficiency. The utilization of

241 formate by microbes as an extra energy source was reported for Candida utilis that can uptake

242 formate in the presence of glucose to increase biomass yield [80]. Meanwhile, similar

243 phenomena were observed in Hansemula polymorpha and Pichia pastoris $[81,82]$. The strategy

244 of employing formate as an extra energy source has been extended to other species that have a

245 formate dehydrogenase $(f d h)$, such as oleaginous yeasts for improving lipid production [83],

246 Penicillium chrysogenum for enhancing penicillin productivity [84], and Bacillus thuringiensis

247 for promoting thuringiensin yield [85]. Further, formate can be generated through an

248 electrochemical process to feed engineered Ralstonia eutropha to produce isobutanol and 3-

249 methyl-1-butanol [86].

Formate $+\mathrm{NAD} \rightarrow \mathrm{CO}_{2}+\mathrm{NADH}$ 
251 On the other side, heterologous expression of an $f d h$ gene enables formate usage in hosts that

252 lack the ability to oxidize formate. For instance, after chromosomal insertion of the $f d h$ gene, $C$.

253 glutamicum was able to utilize formate and produce $20 \%$ more succinate anaerobically in the

254 presence of glucose (formate was used as both NADH and carbon donor) [87]. In yet another

255 case, $f d h$ was introduced into a succinate-producing $E$. coli strain, leading to reduced formate and

256 improved succinate yield [88].

257

258 Current metabolic modeling approach for rational metabolic engineering

259 Metabolic burden has a direct affect on the biochemical productivity of engineered strains

260 and thus, it will be important to explicitly consider metabolic burden during the design process.

261 Numerous good review papers are available to cover different tools and conceptual approaches

262 to model-driven metabolic engineering design (Box 1) including a review by Medema et al. [89]

263 that presents de novo design as involving six steps. The steps include: 1) Pathway prediction (de

264 novo discovery of possible biosynthetic routes), 2) Pathway prioritization (ranking of possible

265 pathways), 3) Metabolic modeling (modeling biochemical function in network context), 4)

266 Pathway selection, 5) Pathway refactoring and integration (molecular-level design specification

267 of necessary DNA constructs), and 6) Product synthesis. These design approaches primarily

268 focus on specifying biochemical pathway usage necessary to produce theoretical yields of a

269 desired product, but experimental strains fall short of theoretical yields due to unaccounted in

270 vivo considerations such as overall metabolic burden.

271

272 Cell-wide genome-scale models for complex cell systems 
Genome-scale models (GSMs) are stoichiometric representations of the biochemical

274 capabilities of a metabolic network based upon the contents of an organism's genome. A key to

275 this approach is establishing gene-protein-reaction (GPR) relationships where identifying a gene

276 in the genome implies the possibility of the associated protein and biochemical reaction.

277 However, if details associated with the molecular aspects of moving from DNA to mRNA and

278 mature protein are absent (as is the case with basic GSMs) then these models allow for any/all

279 reactions to be used as much as needed to fulfill an objective with no restriction meaning that

280 there is no explicit cost to expressing a gene.

281 A substantial amount of work to improve GSMs has been undertaken recently to account

282 for this shortcoming allowing for computational consideration of metabolic costs/burden.

283 Beginning with a careful curation of literature associated with E. coli, an expression matrix (E-

284 Matrix) was developed that included 13,694 reactions associated with all facets of transcription

285 and translation [95] to account for synthesis reactions for DNA, mRNA, proteins, and protein

286 complexes in a sequence-specific manner. The E-Matrix formalism was directly integrated with

287 metabolic networks to create ME (metabolism and expression) models of Thermoto gamaritime

288 [96] and Escherichia coli [97]. The ME models involved extensive attention to molecular detail

289 and formulation of new objective functions for simulations and resulted in improved predictive

290 capabilities including the ability to predict in silico transcriptomes and proteomes [98].

291 In addition to GSMs, a whole-cell model of Mycoplasma genitalium has been generated

292 using 28 modularized subsystems [99]. The approach of this work differed from the development

293 of ME models: in the case of the whole cell Mycoplasma model, different modeling approaches

294 (e.g. ordinary differential equations, Boolean statements, probabilistic, constraint-based) were

295 implemented for the different modules to allow for flexibility to dynamically model each process 
296 in an appropriate way. By developing and integrating modules, it was possible to simulate

297 dynamic whole cell function at a high level of detail. Subsequent to this initial publication, the

298 WholeCellKB database has been established to facilitate development of additional whole cell

299 models using the modularized approach implemented for Mycoplasma [100].

\section{Integration of metabolic burdens of pathway engineering in GSMs}

As metabolic engineering design strategies become more sophisticated and involve

302 implementation of larger DNA constructs, it has become increasingly important to consider the

303 implications of the metabolic burden imposed by expression of synthetic constructs. The

304 approaches discussed above are possible frameworks for implementing a new level of modeling

305 detail that can account for metabolic burden associated with expression and activity of proteins.

306 Improvements to computational modeling frameworks to explicitly include metabolic burden

307 considerations should greatly facilitate metabolic engineering design strategies and help avoid

308 strategies that impose a large non-native metabolic burden. For example, mutants may have

309 decreased productivity due to ATP loss associated with heterologous gene expression. Previous

310 studies have shown that protein synthesis consumes $>60 \%$ of the ATP from cell energy

311 metabolism [101], while protein overexpression is a major metabolic burden in engineered cells

312 [7]. Based upon experimental proteomic studies in E. coli [102], the energetic costs for a single

313 protein synthesis can be calculated to range from about $400-50000$ in terms of high-energy

314 phosphate bond equivalents. The average cost per amino acid ranged from 18 - 38 high-energy

315 phosphate bond equivalents. The unit of phosphate bond equivalents can be related to cellular

316 energy as stored in the phosphate bonds of ATP. Considering protein synthesis costs in terms of

317 phosphate bonds enables a new level of energetic-associated analysis within the framework of

318 GSMs that can predict overall metabolic burden. By explicitly considering the protein production 
319 cost and amino acid composition of individual proteins, metabolic burden associated with

320 enzymes (and their affiliated biochemical reaction) can be analyzed.

321 The use of phosphate bond costs associated with each amino acid provides the means for

322 connecting computational simulations with experimental ${ }^{13} \mathrm{C}-\mathrm{MFA}$ results related to ATP

323 generation and consumption. In addition, FBA may be used to calculate metabolic burdens from

324 synthesis of plasmid DNA (based on sizes and copy numbers). Moreover, ${ }^{13} \mathrm{C}-\mathrm{MFA}$ can be

325 combined to offer comprehensive insights into the intracellular activities responding to the

326 increase in corresponding metabolic reactions [103]. Traditional FBA models lack a precise

327 determination of ATP loss, while ${ }^{13} \mathrm{C}-\mathrm{MFA}$ can provide a quantitative measurement of ATP

328 losses: the ATP maintenance is a sum of the all of the burden in the cell for all the different

329 metabolic functions (protein expression, energy consumption associated with individual

330 reactions, etc.), and thus is a cumulative result of explicitly accounting for burden in individual

331 components of the FBA model. Lastly, RNA profiles can be used with the genome-scale FBA

332 modeling components to provide weighting to expression costs. The integration of high

333 throughput transcriptomics with ${ }^{13} \mathrm{C}-\mathrm{MFA}$ and metabolic models can also be used for analysis of

334 regulatory networks (such as synthetic biology circuits).

\section{Innovative models for assisting synthetic biology applications}

336 First, integrating FBA with process models is important to study scale-up fermentations

337 because large-bioreactors are often dynamic and heterogeneous. These multi-scale models can

338 offer knowledge of intracellular function and overall fermentation performance. For example,

339 dynamic FBA (a combination of kinetic models and FBA) simulates the industrial fermentation

340 of E. coli to produce recombinant proteins [19]. The hybrid of constrained FBA models with

341 agent-based model successfully simulate highly heterogeneous systems (e.g., biofilm) [104]. 
342 Integration of FBA with other information or models will be widely adopted to strengthen the

343 capability of modeling tools and to provide guidance when moving laboratory strains to

344 industrial applications [105].

345 Second, industrial standards play fundamental roles in research \& development and

346 communication within their respective discipline. In the case of synthetic biology, lacking a

347 standard for journal publications hinders fast communication among synthetic biology

348 researchers as well as reproducibility of published work. Thereby, we want to emphasize

349 standardization of publications. For example, the published papers need to clarify their reports

350 on: 1) genetic methodologies; 2) fermentation conditions such as substrate type, incubation

351 mode, nutrient condition, etc.; 3) production titer, rate and yield. For these high profile papers,

352 tracer experiments should be employed to make sure the engineered pathways are actually

353 functional.

354 Third, data-driven models using published synthetic biology papers can provide

355 complementary information to mechanistic based models. For example, a linear empirical model

356 with numerical and categorical variables was generated to predict production yields based on

357 dozens of published papers associated to $E$. coli and $S$. cerevisiae $[8,19]$. That study identified

358 key factors controlling production yields, including biosynthesis steps, metabolic engineering

359 methods, nutrient supplementation, and fermentation conditions. The large number of papers

360 published on metabolic engineering provides a rich database for performing machine learning

361 studies and capture the microbial outputs (production titer, rate, yield) in response to genetic and

362 fermentation conditions. Lessons from the past can allow us to better evaluate the synthetic

363 biology projects. Those published papers can be stored as structural data so that machine

364 learning methods could be applied to extract knowledge for future rational strain development. 
365 Machine learning evolved from pattern recognition, statistics, and optimization, which makes

366 data-driven predictions for outcomes of mutant physiologies [92]. It can assist mechanistic-based

367 GSMs for rational metabolic engineering (Figure 2).

\section{Conclusion}

370 The long-held assumption of never-ending rapid growth in biotechnology in general and

371 synthetic biology in particular has been recently questioned due to lack of substantial return of

372 investment [107]. One of the main reasons of failures in synthetic biology is the largely

373 unaccounted for metabolic burden that may offset synthetic biology benefits and thus careful

374 consideration is necessary at the stage of strain design (see Outstanding Questions). To avoid

375 wrong commercial decisions or investments in those "perpetual-motion-machine" types of

376 projects, ${ }^{13} \mathrm{C}-\mathrm{MFA}$ provides excellent measures and standards for synthetic biology that defines

377 the limitation of synthetic biology, leading to rational metabolic or bioprocess engineering 378 strategies to bypass metabolic burdens (e.g., use of co-cultures or integration of biological 379 conversion with chemical conversions). More importantly, constructing new genome-scale

380 modeling analyses and novel machine learning methods can give broad guidelines for strain 381 development and resolve metabolic unbalancing from synthetic biology applications.

\section{Acknowledgments}

384 Mattheos Koffas would like to acknowledge support from NSF (award MCB 1448657). Yinjie

385 Tang would like to acknowledge support from NSF (DBI 1356669) and the Department of 386 Energy (DESC0012722). 
388 Cipher of Evolutionary Design (CiED): an FBA approach that relies on a genetic algorithm in

389 order to predict gene knockouts for improving fluxes through a metabolic pathway of interest.

390 Dynamic sensor and regulatory system (DSRS): an approach that uses biosensors for 391 intracellular metabolites to regulate metabolic pathways.

392 Flux balance analysis (FBA): a mathematical approach for deriving possible metabolic fluxes in 393 metabolic networks.

394 Genome scale model (GSM): a metabolic network reconstructed based on genome-scale 395 annotations.

396 Growth associated maintenance (GAM): the amount of ATP consumption associated with 397 biosynthesis.

398 Machine learning: a field of study that gives computers the ability to learn from a large amount

399 of experimental data without being explicitly programmed.

400 Metabolic burden: the portion of a host cell's resources - either energy molecules (e.g., $401 \mathrm{NAD}(\mathrm{P}) \mathrm{H}$ and $\mathrm{ATP})$ or carbon building blocks - that is used to construct and operate 402 engineered pathways

403 Metabolic flux analysis (MFA): an analytical method that uses isotopomers to measure in vivo 404 enzyme reaction rates.

405 Non-growth associated maintenance (NGAM): the amount of ATP consumption that does not 406 contribute to biomass synthesis.

407 OptForce, OptStrain series: computational procedures that identify all possible engineering

408 interventions in the metabolic model depending upon whether their flux values must increase,

409 decrease or become equal to zero to meet a pre-specified overproduction target. 
410 Phosphate/Oxygen ratio (P/O ratio): the amount of ATP produced by reduction of an oxygen

411 atom.

412 
415 Figure 1. Biosynthesis yields (g product/g substrate) in engineered E. coli in responses to $\mathrm{P} / \mathrm{O}$

416 ratio, ATP burden from maintenance loss (mmol ATP /g dry cell weight/h), and $\mathrm{O}_{2}$ uptake fluxes

417 (mmol $\mathrm{O}_{2} / \mathrm{g}$ dry cell weight/h). A genome-scale metabolic model (iJO1366) is used for 418 simulations [19].

420 Figure 2. A new modeling paradigm for synthetic biology.

423 Table 1. Summary of experimental metabolic balancing efforts.

\begin{tabular}{|c|c|c|c|c|}
\hline Host & Product & Titer & $\begin{array}{l}\text { Type of Pathway } \\
\text { Optimization }\end{array}$ & Reference \\
\hline E. coli & amorphadiene & $293 \mathrm{mg} / \mathrm{L} / \mathrm{OD} 600$ & $\mathrm{CN}, \mathrm{TS}$ & [30] \\
\hline E. coli & amorphadiene & n.r. & $\mathrm{CN}, \mathrm{TS}$ & [31] \\
\hline E. coli & amorphadiene & $1.6 \mathrm{~g} / \mathrm{L}$ & PTL & [32] \\
\hline E. coli & amorphadiene & $3.6 \mathrm{~g} / \mathrm{L}$ & $\mathrm{TL}$ & [33] \\
\hline S. cerevisiae & amorphadiene & $20 \mathrm{mg} / \mathrm{L}$ & $\mathrm{CP}$ & [34] \\
\hline S. cerevisiae & valencene & $1.5 \mathrm{mg} / \mathrm{L}$ & $\mathrm{CP}$ & [34] \\
\hline E. coli & poly-3-hydroxybutyrate & $70 \% \mathrm{DCW}$ & $\mathrm{CN}$ & [35] \\
\hline E. coli & lycopene & 11,000 p.p.m. & $\mathrm{CN}$ & [35] \\
\hline E. coli & neurosporene & $4.2 \mathrm{mg} / \mathrm{gDCW}$ & TS, TL & [36] \\
\hline E. coli & mevalonate & $740 \mathrm{mg} / \mathrm{L}$ & PTL & [37] \\
\hline E. coli & glucaric acid & $1.7 \mathrm{~g} / \mathrm{L}$ & PTL & [37] \\
\hline E. coli & glucaric acid & $2.5 \mathrm{~g} / \mathrm{L}$ & PTL & {$[38]$} \\
\hline E. coli & glucaric acid & $1.2 \mathrm{~g} / \mathrm{L}$ & DB & [39] \\
\hline E. coli & cis,cismuconic acid & $2 \mathrm{~g} / \mathrm{L}$ & SR & [40] \\
\hline E. coli & cis,cismuconic acid & $4.7 \mathrm{~g} / \mathrm{L}$ & SR & [41] \\
\hline E. coli & 4-hydroxybenzoic acid & $2.3 \mathrm{~g} / \mathrm{L}$ & SR & [41] \\
\hline E. coli & myo-inositol & $1.31 \mathrm{~g} / \mathrm{L}$ & DB & [42] \\
\hline E. coli & riboflavin & $2.70 \mathrm{~g} / \mathrm{L}$ & TL & [43] \\
\hline A. nidulans & penicillin & n.r. & $\mathrm{CP}$ & [44] \\
\hline
\end{tabular}




\begin{tabular}{|c|c|c|c|c|}
\hline S. cerevisiae & xylose utilization & n.a. & $\mathrm{TS}$ & [45] \\
\hline E. coli & chondroitin & $2.4 \mathrm{~g} / \mathrm{L}$ & TS & [46] \\
\hline E. coli & violacein & $1.83 \mathrm{~g} / \mathrm{L}$ & TS & [47] \\
\hline S. cerevisiae & violacein and derivatives & n.r. & TS & [48] \\
\hline E. coli & (+)-catechin & $911 \mathrm{mg} / \mathrm{L}$ & CN, PTL & [49] \\
\hline E. coli & caffeic acid & $3.8 \mathrm{~g} / \mathrm{L}$ & TS & {$[50]$} \\
\hline E. coli & caffeic acid & $106 \mathrm{mg} / \mathrm{L}$ & $\mathrm{CN}$ & [51] \\
\hline E. coli & resveratrol & $35 \mathrm{mg} / \mathrm{L}$ & $\mathrm{CN}, \mathrm{TS}$ & {$[52]$} \\
\hline $\begin{array}{c}\text { E. coli and } S . \\
\text { cerevisiae }\end{array}$ & oxygenated taxanes & $33 \mathrm{mg} / \mathrm{L}$ & SR & [53] \\
\hline E. coli & taxadiene & $1.02 \pm 0.08 \mathrm{~g} / \mathrm{L}$ & $\mathrm{CN}, \mathrm{TS}$ & [54] \\
\hline E. coli & fatty acids & $8.6 \mathrm{~g} / \mathrm{L}$ & $\mathrm{CN}, \mathrm{TL}$ & [55] \\
\hline E. coli & fatty acids & $3.9 \mathrm{~g} / \mathrm{L}$ & DB & [56] \\
\hline E. coli & fatty acid ethyl esters & $1.5 \mathrm{~g} / \mathrm{L}$ & DB & [57] \\
\hline E. coli & butyrate & $7.2 \mathrm{~g} / \mathrm{L}$ & PTL & [58] \\
\hline E. coli & butanol & $6.2 \mathrm{~g} / \mathrm{L}$ & SR & [59] \\
\hline S. cerevisiae & isobutanol & $635 \mathrm{mg} / \mathrm{L}$ & $\mathrm{CP}$ & [60] \\
\hline S. cerevisiae & isopentanol & $95 \mathrm{mg} / \mathrm{L}$ & $\mathrm{CP}$ & [60] \\
\hline S. cerevisiae & 2-methyl-1-butanol & $118 \mathrm{mg} / \mathrm{L}$ & $\mathrm{CP}$ & [60] \\
\hline
\end{tabular}

424 CN, DNA copy number optimization; TS, transcriptional optimization; TL, translational

425 optimization; PTL, post-translational optimization; DB, dynamic balancing; CP,

426 compartmentalization; SR, co-culture strain ratio optimization; n.r., not reported; n.a., not

427 applicable. 


\section{Box 1. Rational algorithmic design}

In this overall framework, some of the most heavily developed work has focused on the

430 metabolic modeling component that is directly tied to metabolic burden. Metabolic control

431 analysis (MCA) is the earliest approach that quantified the correlations between the flux through

432 a pathway and the kinetics of the constituent enzymes. MCA can describe the different levels of

433 flux regulation through a single pathway, but not yet for the distribution of flux through the

434 entire network [21]. On the other hand, genome-scale models (GSMs) used in conjunction with

435 flux balance analysis constitute the most commonly used approach to estimate optimal growth

436 rates and product yields from different feedstocks and the lethality of gene knockouts. Such

437 GSMs can identify the best biosynthesis pathways through elementary mode analysis [90] or

438 systematic pathway modifications (e.g. OptKnock [23,24] and OptStrain [25]).

439 A methodology for predicting optimal metabolic landscapes for the production of a

440 desired metabolite termed Cipher of Evolutionary Design, CiED [91] was initially developed by

441 integrating an evolutionary algorithm around constraint-based modeling, allowing one to

442 simultaneously search an unlimited number of gene targets for modifications. Furthermore,

443 another constraint-based modeling approach termed OptForce [91-94] was utilized to identify

444 genetic perturbations leading to increases in intracellular malonyl-CoA. OptForce suggested the

445 up-regulation of glycolytic reactions, namely glyceraldehyde-3-phosphate dehydrogenase and

446 phosphoglycerate kinase as well as up-regulation of pyruvate dehydrogenase and acetyl-CoA

447 carboxylase. It also suggested down-regulation of reactions in the citric acid cycle, namely

448 malate dehydrogenase, fumarase and aconitase in order to reduce the drain of carbon towards

449 TCA cycle products. OptForce also suggested reducing the activity of TCA reactions instead of 450 completely eliminating them. 
4531 Smolke, C.D. (2009) Building outside of the box: iGEM and the BioBricks Foundation. $454 \quad$ Nat. Biotechnol. 27, 1099-1102

4552 Brown, J. (2007) The iGEM competition: building with biology. IET Synthetic Biology 1, 456 3-6

4573 Woolston, B.M. et al. (2013) Metabolic engineering: past and future. Annu. Rev. Chem. Biomol. Eng. 4, 259-288

4594 Lee, S.Y. and Kim, H.U. (2015) Systems strategies for developing industrial microbial strains. Nat. Biotechnol. 33, 1061-1072 Van Dien, S. (2013) From the first drop to the first truckload: commercialization of microbial processes for renewable chemicals. Curr. Opin. Biotechnol. 24, 1061-1068 Hayden, E.C. (2015) Synthetic biology called to order: meeting launches effort to develop standards for fast-moving field. Nature 520, 141-142

7 Glick, B.R. (1995) Metabolic load and heterologous gene expression. Biotechnol. Adv. 13, 247-261

8 Colletti, P.F. et al. (2011) Evaluating factors that influence microbial synthesis yields by linear regression with numerical and ordinal variables. Biotechnol. Bioeng. 108, 893-901

9 Poust, S. et al. (2014) Narrowing the gap between the promise and reality of polyketide synthases as a synthetic biology platform. Curr. Opin. Biotechnol. 30, 32-39

10 Glick, B.R. et al. (1985) Physiological effects of plasmid DNA transformation on Azotobacter vinelandii. Can. J. Microbiol. 32, 145-148

11 Hong, Y. et al. (1991) Biological consequences of plasmid transformation of the plant growth promoting rhizobacterium Paseudomonas putida GR12-2. Can. J. Microbiol. 37, 796-799

12 Phillips, R. and Milo, R. (2009) A feeling for the numbers in biology. Proc. Natl. Acad. Sci. U.S.A. 106, 21465-21471

13 Zhuang, K. et al. (2011) Economics of membrane occupancy and respiro-fermentation. Mol. Syst. Biol. 7, 500-508

14 Chen, X. et al. (2011) Synergy between ${ }^{13}$ C-metabolic flux analysis and flux balance analysis for understanding metabolic adaptation to anaerobiosis in E. coli. Metab. Eng. 13, $38-48$

15 Wu, S.G. et al. (2015) An ancient Chinese wisdom for metabolic engineering: Yin-Yang. Microb. Cell. Fact. 14, 39-47

16 Sauer, U. and Bailey, J.E. (1999) Estimation of P-to-O ratio in Bacillus subtilis and its influence on maximum ribofavin yield. Biotechnol. Bioeng. 64, 750-754

17 Birnbaum, S. and Bailey, J.E. (1991) Plasmid presence changes the relative levels of many host cell proteins and ribosome components in recombinant Escherichia coli. Biotechnol. Bioeng. 37, 736-745

18 Varma, A. and Palsson, B.O. (1994) Stoichiometric flux balance models quantitatively predict growth and metabolic by-product secretion in wild-type Escheichia coli W3110. Appl. Environ. Microb. 60, 3724-3731

19 Varman, A.M. et al. (2011) Statistics-based model for prediction of chemical biosynthesis yield from Saccharomyces cerevisiae. Microb. Cell. Fact. 10, 45-56 
20 Heyland, J. et al. (2011) Quantification of metabolic limitations during recombinant protein production in Escherichia coli. J. Biotechnol. 155, 178-184

21 Sauer, U. (2006) Metabolic networks in motion: ${ }^{13}$ C-based flux analysis. Mol. Syst. Biol. 2 , 62

22 He, L. et al. (2014) Central metabolic responses to the overproduction of fatty acids Escherichia coli based on ${ }^{13} \mathrm{C}$-metabolic flux analysis. Biotechnol. Bioeng. 111, 575-585

23 Buescher, J.M. et al. (2015) A roadmap for interpreting ${ }^{13} \mathrm{C}$ metabolite labeling patterns

502

503

504

505

506

507

508

509

510

511

512

513

514

515

516

517

518

519

520

521

522

523

524

525

526

527

528

529

530

531

532

533

534

535

536

537

538

539 from cells. Curr. Opin. Biotechnol. 34, 189-201

24 Martin, H.G. et al. (2015) A method to constrain genome-scale models with ${ }^{13} \mathrm{C}$ labeling data. PLoS Comput. Biol. 11, e1004363-e1004396

25 Gopalakrishnan, S. and Maranas, C.D. (2015) ${ }^{13} \mathrm{C}$ metabolic flux analysis at a genomescale. Metab. Eng. 32, 12-22

26 Hua, Q. et al. (2007) Metabolic characterization of Escherichia coli strains adapted to growth on lactate. Appl. Environ. Microb. 73, 4639-4647

27 Perrenoud, A. and Sauer, U. (2005) Impact of global transcriptional regulation by ArcA, ArcB, Cra, Crp, Cya, Fnr, and Mlc on glucose catabolism in Escherichia coli. J. Bacteriol. 187, 3171-3179

28 Kim, S.M. et al. (2015) Simultaneous utilization of glucose and xylose via novel mechanisms in engineered Escherichia coli. Metab. Eng. 30, 141-148

29 Smolke, C. and Keasling, J. (2002) Effect of copy number and mRNA processing and stabilization on transcript and protein levels from an engineered dual-gene operon. Biotechnol. Bioeng. 78, 412-424

30 Anthony, J.R. et al. (2009) Optimization of the mevalonate-based isoprenoid biosynthetic pathway in Escherichia coli for production of the anti-malarial drug precursor amorpha4,11-diene. Metab. Eng. 11, 13-19

31 Pitera, D.J. et al. (2007) Balancing a heterologous mevalonate pathway for improved isoprenoid production in Escherichia coli. Metab. Eng. 9, 193-207

32 Dahl, R.H. et al. (2013) Engineering dynamic pathway regulation using stress-response promoters. Nat. Biotechnol. 31, 1039-1046

33 Nowroozi, F.F. et al. (2014) Metabolic pathway optimization using ribosome binding site variants and combinatorial gene assembly. Appl. Microbiol. Biotechnol. 98, 1567-1581

34 Farhi, M. et al. (2011) Harnessing yeast subcellular compartments for the production of plant terpenoids. Metab. Eng. 13, 474-481

35 Tyo, K.E. et al. (2009) Stabilized gene duplication enables long-term selection-free heterologous pathway expression. Nat. Biotechnol. 27, 760-765

$36 \mathrm{Ng}, \mathrm{C} . Y$. et al. (2015) Rational design of a synthetic Entner-Doudoroff pathway for improved and controllable NADPH regeneration. Metab. Eng. 29, 86-96

37 Dueber, J.E. et al. (2009) Synthetic protein scaffolds provide modular control over metabolic flux. Nat. Biotechnol. 27, 753-759

38 Moon, T.S. et al. (2010) Use of modular, synthetic scaffolds for improved production of glucaric acid in engineered E. coli. Metab. Eng. 12, 298-305

39 Reizman, I.M. et al. (2015) Improvement of glucaric acid production in via dynamic control of metabolic fluxes. Metab. Eng. Commun. 2, 109-116

40 Zhang, H. et al. (2015) Engineering E. coli-E. coli cocultures for production of muconic acid from glycerol. Microb. Cell. Fact. 14, 134 
54041 Zhang, H. et al. (2015) Engineering Escherichia coli coculture systems for the production of biochemical products. Proc. Natl. Acad. Sci. U.S.A. 112, 8266-8271

54242 Brockman, I.M. and Prather, K.L. (2015) Dynamic knockdown of E. coli central metabolism for redirecting fluxes of primary metabolites. Metab. Eng. 28, 104-113

43 Lin, Z. et al. (2014) Metabolic engineering of Escherichia coli for the production of riboflavin. Microb. Cell. Fact. 13, 104-115

44 Herr, A. and Fischer, R. (2014) Improvement of Aspergillus nidulans penicillin production by targeting AcvA to peroxisomes. Metab. Eng. 25, 131-139

45 Latimer, L.N. et al. (2014) Employing a combinatorial expression approach to characterize xylose utilization in Saccharomyces cerevisiae. Metab. Eng. 25, 20-29

46 He, W. et al. (2015) Production of chondroitin in metabolically engineered E. coli. Metab. Eng. 27, 92-100

47 Jones, J.A. et al. (2015) ePathOptimize: A combinatorial approach for transcriptional balancing of metabolic pathways. Sci. Rep. 5, 11301-11310

48 Lee, M.E. et al. (2013) Expression-level optimization of a multi-enzyme pathway in the absence of a high-throughput assay. Nucleic Acids Res. 41, 10668-10678

49 Zhao, S. et al. (2015) Improvement of catechin production in Escherichia coli through combinatorial metabolic engineering. Metab. Eng. 28, 43-53

50 Huang, Q. et al. (2013) Caffeic acid production enhancement by engineering a phenylalanine over-producing Escherichia coli strain. Biotechnol. Bioeng. 110, 3188-3196

51 Zhang, H. and Stephanopoulos, G. (2013) Engineering E. coli for caffeic acid biosynthesis from renewable sugars. Appl. Microbiol. Biotechnol. 97, 3333-3341

$52 \mathrm{Wu}$, J. et al. (2013) Multivariate modular metabolic engineering of Escherichia coli to produce resveratrol from L-tyrosine. J. Biotechnol. 167, 404-411

53 Zhou, K. et al. (2015) Distributing a metabolic pathway among a microbial consortium enhances production of natural products. Nat. Biotechnol. 33, 377-383

54 Ajikumar, P.K. et al. (2010) Isoprenoid pathway optimization for taxol precursor overproduction in Escherichia coli. Science 330, 70-74

$55 \mathrm{Xu}$, P. et al. (2013) Modular optimization of multi-gene pathways for fatty acids production in E. coli. Nat. Commun. 4, 1409-1416

$56 \mathrm{Xu}, \mathrm{P}$. et al. (2014) Improving fatty acids production by engineering dynamic pathway regulation and metabolic control. Proc. Natl. Acad. Sci. U. S. A. 111, 11299-11304

57 Zhang, F. et al. (2012) Design of a dynamic sensor-regulator system for production of chemicals and fuels derived from fatty acids. Nat. Biotechnol. 30, 354-359

58 Baek, J.-M. et al. (2013) Butyrate production in engineered Escherichia coli with synthetic scaffolds. Biotechnol. Bioeng. 110, 2790-2794

59 Saini, M. et al. (2015) Potential production platform of n-butanol in Escherichia coli. Metab. Eng. 27, 76-82

60 Avalos, J.L. et al. (2013) Compartmentalization of metabolic pathways in yeast mitochondria improves the production of branched-chain alcohols. Nat. Biotechnol. 31, 335-341

$61 \mathrm{Xu}$, P. et al. (2012) ePathBrick: a synthetic biology platform for engineering metabolic pathways in E. coli. ACS Synth. Biol. 1, 256-266

62 Cress, B.F. et al. (2015) CRISPathBrick: modular combinatorial assembly of type II-A CRISPR arrays for dCas9-mediated multiplex transcriptional repression in E. coli. ACS Synth. Biol. 4, 987-1000 
63 Gibson, D. et al. (2010) Creation of a bacterial cell controlled by a chemically synthesized genome. Science 329, 52-56

588

64 Forster, A.C. and Church, G.M. (2006) Towards synthesis of a minimal cell. Mol. Syst. Biol. 2, 45-54

65 Trinh, C.T. et al. (2008) Minimal Escherichia coli cell for the most efficient production of ethanol from hexoses and pentoses. Appl. Environ. Microb. 74, 3634-3643

593

594

595

66 Westers, H. et al. (2003) Genome engineering reveals large dispensable regions in Bacillus subtilis. Mol. Biol. Evol. 20, 2076-2090

67 Eames, M. and Kortemme, T. (2012) Cost-benefit tradeoffs in engineered lac operons. Science 336, 911-915

68 Khosla, C. et al. (1990) Expression of intracellular hemoglobin improves protein synthesis in oxygen-limited Escherichia coli. Nat. Biotechnol. 8, 849-853

69 Zamboni, N. et al. (2003) Reducing maintenance metabolism by metabolic engineering of respiration improves riboflavin production by Bacillus subtilis. Metab. Eng. 5, 49-55

70 Liu, Y. et al. (2014) Spatial modulation of key pathway enzymes by DNA-guided scaffold system and respiration chain engineering for improved $\mathrm{N}$-acetylglucosamine production by Bacillus subtilis. Metab. Eng. 24, 61-69

71 Kabus, A. et al. (2007) Role of cytochrome bd oxidase from Corynebacterium glutamicum in growth and lysine production. Appl. Environ. Microb. 73, 861-868

72 Kabashima, Y. et al. (2009) Correlation between proton translocation and growth: genetic analysis of the respiratory chain of Corynebacterium glutamicum. J. Biochem. 146, 845855

73 Wijffels, R.H. et al. (2013) Potential of industrial biotechnology with cyanobacteria and eukaryotic microalgae. Curr. Opin. Biotechnol. 24, 405-413

74 You, L. et al. (2015) Photoheterotrophic fluxome in Synechocystis sp. strain PCC 6803 and its implications for cyanobacterial bioenergetics. J. Bacteriol. 197, 943-950

75 Walter, J.M. et al. (2007) Light-powering Escherichia coli with proteorhodopsin. Proc. Natl. Acad. Sci. U.S.A. 104, 2408-2412

76 Martinez, A. et al. (2007) Proteorhodopsin photosystem gene expression enables photophosphorylation in a heterologous host. Proc. Natl. Acad. Sci. U.S.A. 104, 5590-5595

77 Steindler, L. et al. (2011) Energy starved Candidatus Pelagibacter ubique substitutes lightmediated ATP production for endogenous carbon respiration. PloS One 6, e19725-e19734

78 Fuhrman, J.A. et al. (2008) Proteorhodopsins: an array of physiological roles? Nat. Rev. Microbiol. 6, 488-494

79 Johnson, E.T. and Schmidt-Dannert, C. (2008) Light-energy conversion in engineered microorganisms. Trends Biotechnol. 26, 682-689

80 Bruinenberg, P.M. et al. (1983) An enzyme analysis of NADPH production and consumption in Candida utilis. J. Gen. Appl. Microbiol. 129, 965-971

81 Babel, W. et al. (1983) Improvement of growth yield of yeast on glucose to the maximum by using an additional energy source. Arch. Microbial. 136, 203-208

82 Hazeu, W. and Donker, R. (1983) A continuous culture study of methanol and formate utilization by the yeast Piohia Pastoris. Biotechnol. Lett. 5, 399-404

83 Lian, J. et al. (2012) Yeast fermentation of carboxylic acids obtained from pyrolytic aqueous phases for lipid production. Bioresour. Technol. 118, 177-186 
84 Harris, D.M. et al. (2007) Formate as an auxiliary substrate for glucose-limited cultivation of Penicillium chrysogenum: impact on penicillin G production and biomass yield. Appl. Environ. Microb. 73, 5020-5025

85 Zhi, W. et al. (2007) A fundamental regulatory role of formate on thuringiensin production by resting cell of Bacillus thuringiensis YBT-032. Bioprocess. Biosyst. Eng. 30, 225-229

$86 \mathrm{Li}, \mathrm{H}$. et al. (2012) Integrated electromicrobial conversion of $\mathrm{CO}_{2}$ to higher alcohols. Science 335, 1596-1597

87 Litsanov, B. et al. (2012) Toward homosuccinate fermentation: metabolic engineering of Corynebacterium glutamicum for anaerobic production of succinate from glucose and formate. Appl. Environ. Microb. 78, 3325-3337

88 Balzer, G.J. et al. (2013) Metabolic engineering of Escherichia coli to minimize byproduct formate and improving succinate productivity through increasing NADH availability by heterologous expression of $\mathrm{NAD}^{+}$-dependent formate dehydrogenase. Metab. Eng. 20, 1-8

89 Medema, M.H. et al. (2012) Computational tools for the synthetic design of biochemical pathways. Nat. Rev. Microbiol. 10, 191-202

90 Trinh, C.T. et al. (2011) Redesigning Escherichia coli metabolism for anaerobic production of isobutanol. Appl. Environ. Microb. 77, 4894-4904

91 Fowler, Z.L. et al. (2009) Increased malonyl coenzyme A biosynthesis by tuning the Escherichia coli metabolic network and its application to flavanone production. Appl. Environ. Microb. 75, 5831-5839

92 Chemler, J.A. et al. (2010) Improving NADPH availability for natural product biosynthesis in Escherichia coli by metabolic engineering. Metab. Eng. 12, 96-104

93 Bhan, N. et al. (2013) Redirecting carbon flux into malonyl-CoA to improve resveratrol titers: Proof of concept for genetic interventions predicted by OptForce computational framework. Chem. Eng. Sci. 103, 109-114

94 Ranganathan, S. et al. (2010) OptForce: an optimization procedure for identifying all genetic manipulations leading to targeted overproductions. PLoS Comput. Biol. 6, e1000744-e1000754

95 Thiele, I. et al. (2009) Genome-scale reconstruction of Escherichia coli's transcriptional and translational machinery: a knowledge base, its mathematical formulation, and its functional characterization. PLoS Comput. Biol. 5, e1000312-e1000324

96 Lerman, J.A. et al. (2012) In silico method for modelling metabolism and gene product expression at genome scale. Nat. Commun. 3, 929-938

97 Thiele, I. et al. (2012) Multiscale modeling of metabolism and macromolecular synthesis in E. coli and its application to the evolution of codon usage. PLoS One 7, e45635-e45652

98 O'Brien, E.J. et al. (2013) Genome-scale models of metabolism and gene expression extend and refine growth phenotype prediction. Mol. Syst. Biol. 9, 693-705

99 Karr, J.R. et al. (2012) A whole-cell computational model predicts phenotype from genotype. Cell 150, 389-401

100 Karr, J.R. et al. (2013) WholeCellKB: model organism databases for comprehensive whole-cell models. Nucleic Acids Res. 41, D787-D792

101 Russell, J.B. and Cook, G.M. (1995) Energetics of bacterial growth: balance of anabolic and catalolic reactions. Microbiological Review 59(1), 48-62

102 Akashi, H. and Gojobori, T. (2002) Metabolic efficiency and amino acid composition in the proteomes of Escherichia coli and Bacillus subtilis. Proc. Natl. Acad. Sci. U.S.A. 99, $3695-3700$ 
676103 Pharkya, P. et al. (2004) Optstrain: a computational framework for redesign of microbial 677 production systems. Genome Res. 14, 2367-2376

678104 Biggs, M.B. and Papin, J.A. (2013) Novel multiscale modeling tool applied to 679 

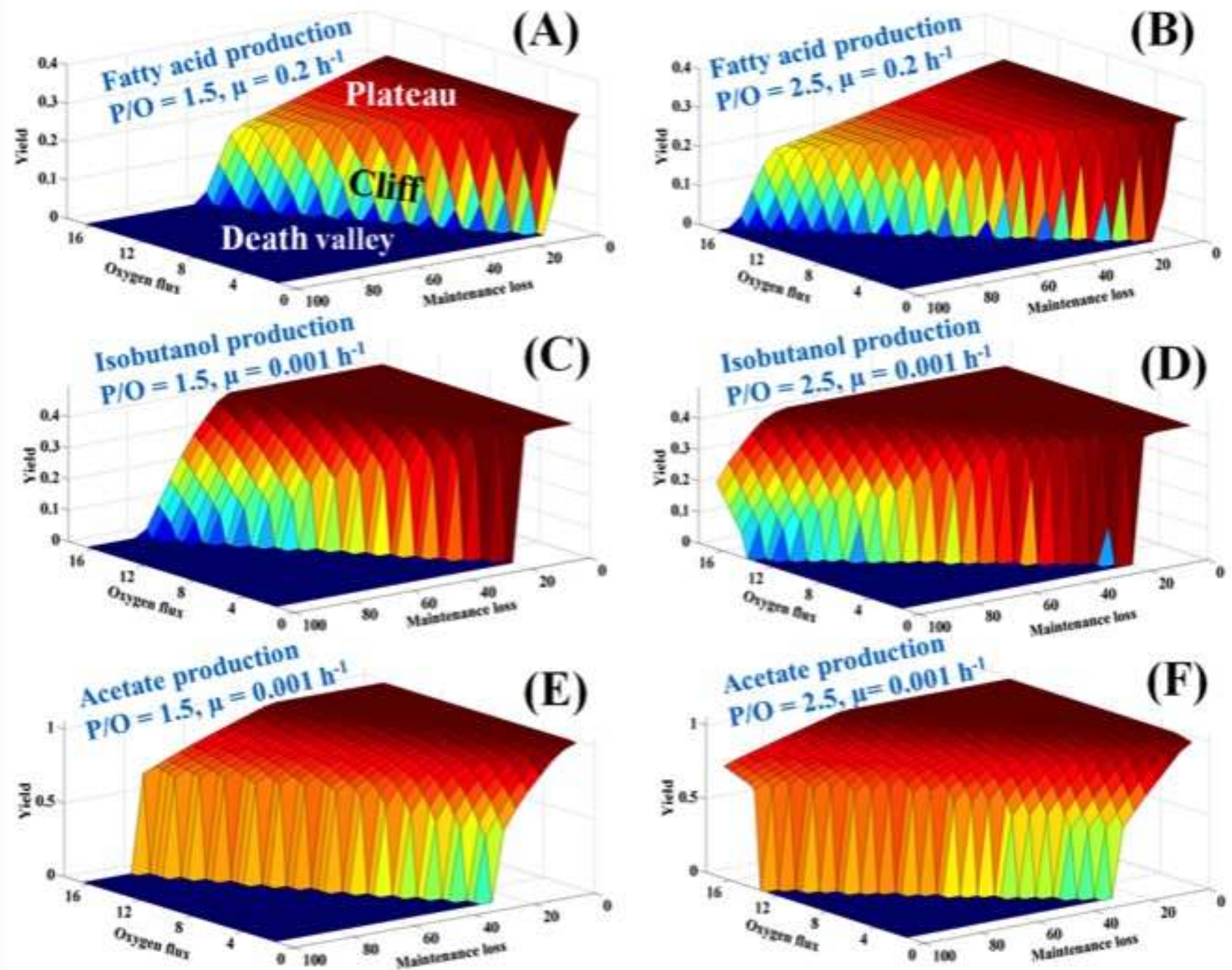


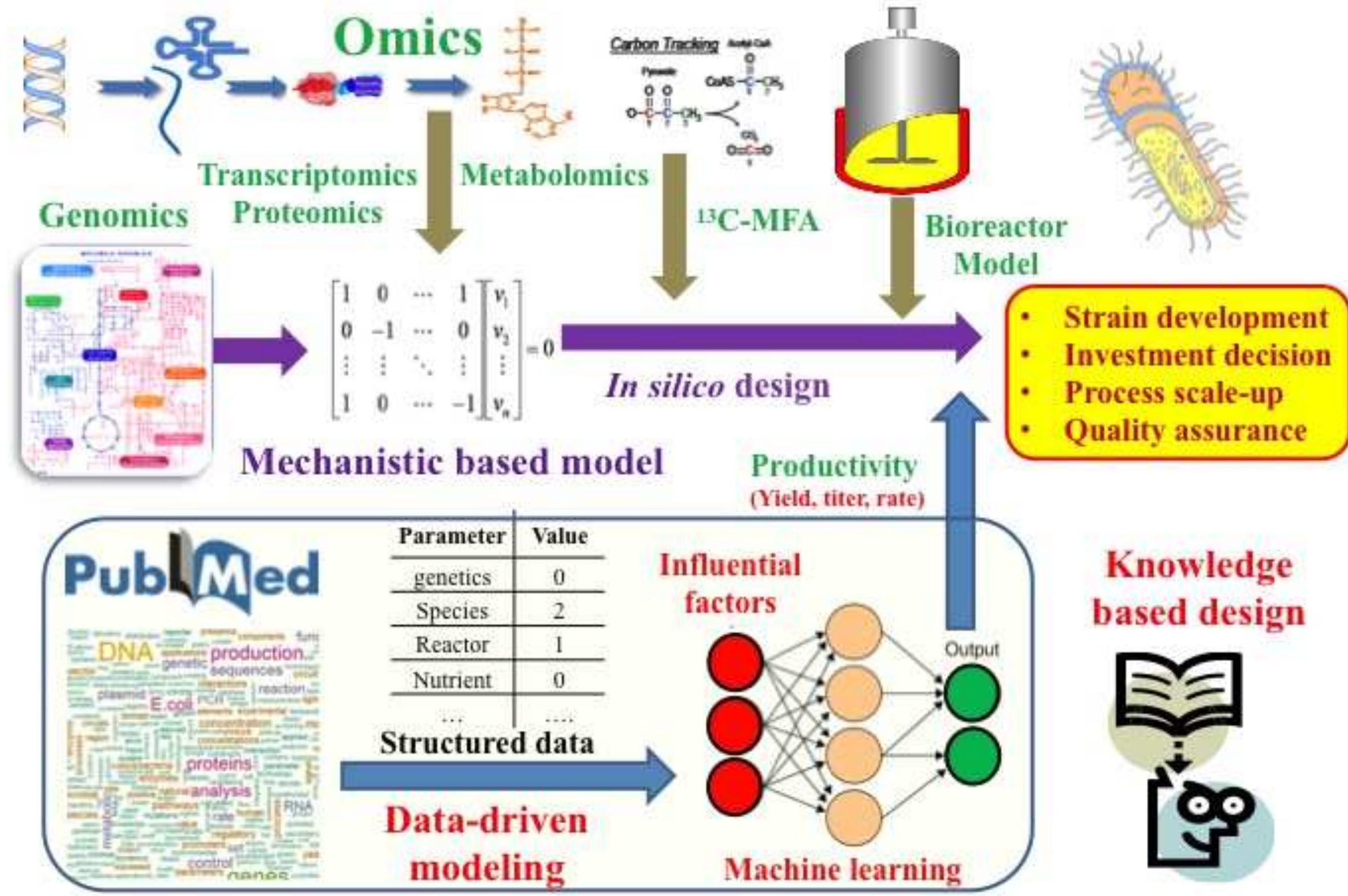

